\title{
Effectiveness of jejunostomy for enteral nutrition during complete thoracoscopic and laparoscopic Ivor-Lewis esophagectomy in thoracic segment esophageal carcinoma
}

\author{
Jieyong Tian*, Xinyu Mei, Mingfa Guo, Ran Xiong and Xiangxiang Sun
}

\begin{abstract}
Background: Although jejunostomy is widely used in complete thoracoscopic and laparoscopic minimally invasive Ivor-Lewis esophagectomy, its clinical effectiveness remains undefined. This study aimed to assess the therapeutic and side effects of jejunostomy in patients undergoing Ivor-Lewis esophagectomy for thoracic segment esophageal carcinoma.

Methods: A total of 1400 patients with esophageal carcinoma who underwent minimally invasive esophagectomy in the Thoracic Surgery of our hospital from 2015 to 2018 were retrospectively evaluated. Of these, 356 and 1044 were treated with nasojejunal feeding tubes (Nasojejunal group) and by jejunostomy (Jejunostomy group), respectively. Clinicopathologic factors, postoperative complications and tubule-related complications between the two groups were compared.

Results: Both groups were well-balanced for clinicopathological data, except tumor location, which was significantly different $(P<0.001)$. Operation time $(208.8 \pm 53.5 \mathrm{~min}$ vs. $218.1 \pm 43.2 \mathrm{~min})$ was shorter in the Jejunostomy group compared with the Nasojejunal group, while intraoperative ( $26.6 \pm 10.4$ min vs $18.4 \pm 9.1 \mathrm{~min}$ ) and postoperative $(38.6 \pm 6.9 \mathrm{~min}$ vs $18.5 \pm 7.6 \mathrm{~min}$ ) indwelling times of nutrition tubes were prolonged (all $P<0.05)$. Postoperative pulmonary infection (17.0\% vs $22.2 \%)$, incision infection $(0.2 \%$ vs $1.1 \%)$, nutrient tube slippage $(0.2 \%$ vs $5.1 \%)$ and nutrient reflux $1(0.1 \%$ vs $5.6 \%)$ rates were reduced in the Jejunostomy group compared with the Nasojejunal group $(P<0.05)$. Meanwhile, ileus rates perioperatively $(1.7 \%$ vs $0.3 \%)$ and at 3 postoperative months $(1.7 \%$ vs $0.3 \%)$ were both higher in the Jejunostomy group compared with the Nasojejunal group.

Conclusions: Jejunostomy is a reliable enteral nutrition method in Ivor-Lewis esophagectomy for thoracic segment esophageal carcinoma.
\end{abstract}

Keywords: Esophageal neoplasm, Esophagectomy, Enteral nutrition, Jejunostomy, Nasointestinal tube, Ivor-Lewis, McKowen

\footnotetext{
* Correspondence: tjyong77@sina.cn

Department of Thoracic Surgery, The First Affiliated Hospital of USTC,

Division of Life Sciences and Medicine, University of Science and Technology of China, Hefei 230036, Anhui, China
}

C C The Author(s). 2020 Open Access This article is licensed under a Creative Commons Attribution 4.0 International License, which permits use, sharing, adaptation, distribution and reproduction in any medium or format, as long as you give appropriate credit to the original author(s) and the source, provide a link to the Creative Commons licence, and indicate if changes were made. The images or other third party material in this article are included in the article's Creative Commons licence, unless indicated otherwise in a credit line to the material. If material is not included in the article's Creative Commons licence and your intended use is not permitted by statutory regulation or exceeds the permitted use, you will need to obtain permission directly from the copyright holder. To view a copy of this licence, visit http://creativecommons.org/licenses/by/4.0/ The Creative Commons Public Domain Dedication waiver (http://creativecommons.org/publicdomain/zero/1.0/) applies to the data made available in this article, unless otherwise stated in a credit line to the data. 


\section{Background}

Esophageal cancer, an extremely aggressive malignancy, ranks numbers 6 and 8 among the deadliest and most common cancers worldwide, respectively; its incidence exceeds 100 cases/100000 person-years in some regions, with a 5-year survival rate approximating 15-25\% [1]. Esophageal cancer can be divided into squamous cell carcinoma and adenocarcinoma subtypes [2]. Risk factors include gender, race, smoking, drinking, diet, genetics, obesity, drug use, and a history of mediastinal radiation, gastroesophageal reflux disease or Barrett's esophagus [1, 3].

At present, surgery is the main treatment option for resectable esophageal carcinoma [4, 5]. The National Comprehensive Cancer Network (NCCN) provides guidelines for treating esophageal cancer, and surgical options encompass local mucosal resection and ablation therapies and esophagectomy [6]. Postoperatively, the incidence of anemia and hypoproteinemia are high, because patients usually have long-term eating difficulties, especially the elderly [7]. Therefore, the postoperative nutritional status of patients with esophageal carcinoma is considered an important factor in preventing postoperative complications and ensuring the success of surgical treatment $[8,9]$.

Given its advantages of comprehensive nutrition, easy initiation, and satisfying physiological requirements for nutrient absorption in the human body, enteral nutrition (EN) has been applied by most clinicians [10-12]. Currently, the most common EN methods employ nasointestinal and jejunostomy tubes. Clinical application of the nasointestinal tube is common, and its efficacy is widely recognized, but there are shortcomings, including poor comfort and easy slippage during tube indwelling, as well as poor tolerance [13]. Jejunostomy is mainly used in complete thoracoscopic and laparoscopic minimally invasive Ivor-Lewis esophagectomy, which can significantly improve the patients' degree of comfort and portability due to the nutrition tube being located in the abdomen, thus improving the quality of life during indwelling and in postoperative EN support [14]. However, some scholars believe that jejunostomy is an invasive operation, which increases surgical difficulty and trauma, with more tubule-related complications [15].

Since January 2015, our center has routinely used laparoscopic jejunostomy for minimally invasive IvorLewis esophagectomy, and routine intraoperative indwelling of nasointestinal tubes for minimally invasive McKowen esophagectomy. Despite the wide application of jejunostomy, its clinical effectiveness remains undefined. Therefore, the present retrospective study aimed to assess the therapeutic and undesired effects of jejunostomy in individuals administered Ivor-Lewis esophagectomy for thoracic segment esophageal carcinoma. The clinical data of 1400 patients with esophageal carcinoma were assessed, and various parameters in both EN methods were compared to investigate the effectiveness and reliability of jejunostomy in complete thoracoscopic and laparoscopic minimally invasive Ivor-Lewis esophagectomy.

\section{Methods}

\section{Study design and patients}

This was a retrospective study conducted at the department of Thoracic Surgery, the First Affiliated Hospital of University of Science and Technology of China, from January 2015 to June 2018. The surgical approach was usually chosen according to tumor location. Tumor staging in esophageal cancer patients was performed using the AJCC 8th edition of TNM staging system [16]. Cases were consecutively enrolled in the study and those with upper esophageal cancer usually underwent McKeown surgery, while those with middle or lower esophageal cancer could undergo either Ivor-Lewis or McKeown surgery. The patients undergoing minimally invasive Ivor-Lewis esophagectomy and McKeown surgery received routine tube feeding via jejunostomy and indwelling nasointestinal tubes, respectively. Accordingly, the patients were assigned to the jejunostomy and nasojejunal groups (Fig. 1).

Inclusion criteria were: 1) confirmed esophageal carcinoma by postoperative pathology; 2) minimally invasive esophagectomy (Ivor-Lewis or McKowen surgery); and 3) intraoperative indwelling of nasointestinal or jejunostomy tube. Exclusion criteria were: 1) incomplete clinical data; 2) conversion to open surgery intraoperatively; or 3) postoperative parenteral nutrition.

\section{Surgical methods}

Patients underwent McKeown surgery to achieve esophagogastric anastomosis, as described previously [17]. During surgery, the surgeons placed the nasointestinal tube into the second lateral hole of the end of the gastric tube and introduced it from the nasal cavity, passing through the esophagus, residual stomach, and the pylorus, to reach the duodenum. Then, the surgeons grasped the bifurcation of the gastric tube and the duodenal nutrition tube in the abdominal cavity and removed the nutrition tube until the two were separated. Subsequently, the nutrition tube was further inserted by about $20 \mathrm{~cm}$, while the gastric tube was withdrawn to enter the stomach. Routine indwelling of the nasointestinal tube was performed at an overall depth of about $75-80 \mathrm{~cm}$ away from incisors. After indwelling completion, the nasointestinal tube and the gastric tube were simultaneously fixed to the nose with adhesive plaster and a string. 


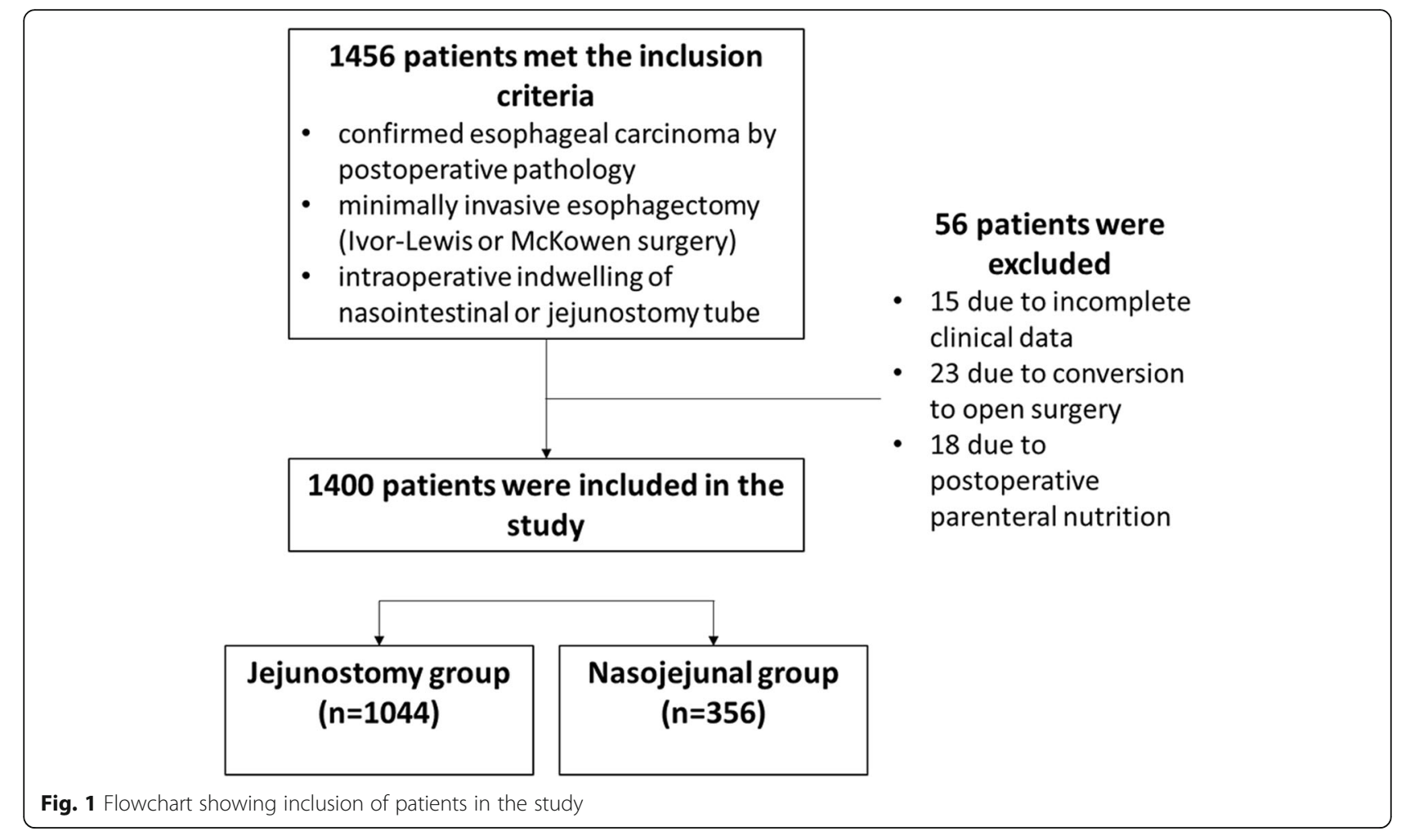

The patients received Ivor-Lewi surgery $[18,19]$ to achieve intrathoracic esophagogastrostomy. After laparoscopic preparation of the tubular stomach and abdominal lymph node dissection, the jejunum was lifted $25 \mathrm{~cm}$ from the ligament of Treitz, and laparoscopic purse string suture was conducted with a 4-0 non-invasive suture; the fixation line was reserved at the area $0.5 \mathrm{~cm}$ away from the proximal end. Using the operating hole at the left lower abdomen as the ostomy hole, cauterization was performed with an electrotome, penetrating the intestinal wall. Then, a Flocare $\mathrm{CH} 10-130$ nutrition tube was placed into the distal end of the jejunum, and water was supplied while the nutrition tube was being placed into the intestinal tube, at a depth of $40 \mathrm{~cm}$. After water injection was smooth, the purse string was tightened. The end of the string was inserted subcutaneously with a needle, and the jejunostomy tube was fixed to the abdominal wall with a No. 4 suture.

All patients in both groups discontinued enteral nutrition 3 weeks after surgery.

\section{Data collection}

General clinical parameters (age, sex, tumor location, pTMN stage, pathological G stage, and previous history of abdominal surgery), perioperative data (surgery time, intraoperative indwelling time, anal exhaust time, postoperative hospital stay, hospitalization expenses, perioperative complications, and tubule-related complications), and postoperative long-term complications were assessed.

The primary outcome was perioperative complications. Postoperative long-term complications, tubule-related complications, postoperative indwelling time of nutrition tube, anal exhaust time, hospitalization expense, and postoperative hospital stay were secondary outcomes.

\section{Follow up}

Outpatient follow up was performed at 1, 3, 6, 9, and 12 months after surgery. Routine blood tests, blood biochemistry, tumor biomarkers, esophagography, and chest and upper abdominal CT examinations were performed. In addition, the patients were evaluated for digestive symptoms, including abdominal pain, diarrhea, vomiting, acid reflux, and/or eating/swallowing obstruction. Patients unable to return to the outpatient center were followed up by phone, recording symptoms and results of examinations performed at local hospitals. The follow up was completed in December 2018.

\section{Statistical analysis}

Data analysis was performed with the SPSS 22.0 statistical software (SPSS, USA). Quantitative data with normal distribution were expressed as mean $\pm S D$ and compared by Student's $t$ test. Categorical data were 
expressed as frequency and percentage and assessed by the $X^{2}$ and Wilcoxon rank sum tests for nominal and ordinal variables, respectively. $P<0.05$ was considered statistically significant.

\section{Results}

\section{Patient characteristics}

A total of 1400 patients were enrolled in the present study. Of these, 1044 underwent minimally invasive Ivor-Lewis surgery, with routine jejunostomy; meanwhile, 356 underwent minimally invasive McKeown surgery, with routine indwelling nasal nutrition tubes. The median follow-up time was 8 months (1-12 months). 384 patients were lost to follow-up.

There were no significant differences in sex, age, preoperative ASA grade, postoperative TNM stage, pathological G stage, and previous history of abdominal surgery between the two groups (all $P>0.05$ ). Regarding tumor location, the difference between the two groups was statistically significant $(P<0.001)$. All patients in the
Jejunostomy group had middle or lower thoracic esophageal carcinoma; in the Nasojejunal group, 201, 147 and 8 patients were upper, middle and lower thoracic esophageal carcinoma cases, respectively. Among them, there were 8 cases of lower thoracic esophageal carcinoma with T1N0M0 complicated with preoperative severe lung function damage, including 5 and 3 administered esophagectomy and inflatable mediastinoscopy plus laparoscopic esophagectomy, respectively (Table 1).

\section{Perioperative data and postoperative long-term outcomes}

Perioperative and postoperative findings are summarized in Table 2. Operation time $(208.8 \pm 53.5 \mathrm{~min}$ vs. $218.1 \pm$ $43.2 \mathrm{~min}$ ) was shorter in the Jejunostomy group compared with the Nasojejunal group, while intraoperative $(26.6 \pm 10.4 \mathrm{~min}$ vs $18.4 \pm 9.1 \mathrm{~min})$ and postoperative (38.6 $\pm 6.9 \mathrm{~min}$ vs $18.5 \pm 7.6 \mathrm{~min})$ indwelling times of nutrition tubes were prolonged (all $P<0.05$ ). There were no significant differences in hospitalization expenses,

Table 1 Patient baseline data

\begin{tabular}{|c|c|c|c|}
\hline & \multicolumn{3}{|l|}{ Before match } \\
\hline & Jejunostomy group $(n=1044)$ & Nasojejunal group $(n=356)$ & $P$ value \\
\hline Sex (Male) & $817(78.3 \%)$ & $272(76.4 \%)$ & 0.514 \\
\hline Age (y) & $61.8 \pm 16.5$ & $63.5 \pm 14.6$ & 0.084 \\
\hline Tumor location & & & $<0.001$ \\
\hline Superior segment & $0(0.0 \%)$ & $201(56.5 \%)$ & \\
\hline Middle segment & $621(59.5 \%)$ & $147(41.3 \%)$ & \\
\hline Inferior segment & $423(40.5 \%)$ & $8(2.2 \%)$ & \\
\hline Preoperative ASA staging & & & 0.085 \\
\hline । & $176(16.9 \%)$ & $53(14.9 \%)$ & \\
\hline$\|$ & 725 (69.4\%) & $237(66.6 \%)$ & \\
\hline III & $143(13.7 \%)$ & $66(18.5 \%)$ & \\
\hline \multicolumn{4}{|l|}{ Postoperative TNM staging } \\
\hline Postoperative T staging & & & 0.172 \\
\hline $\mathrm{T} 1$ & $103(9.8 \%)$ & $35(9.8 \%)$ & \\
\hline $\mathrm{T} 2$ & $216(20.7 \%)$ & $64(18.0 \%)$ & \\
\hline T3 & $624(59.8 \%)$ & $208(58.4 \%)$ & \\
\hline $\mathrm{T} 4$ & $101(9.7 \%)$ & $49(13.8 \%)$ & \\
\hline Postoperative N staging & & & 0.127 \\
\hline No & $208(19.9 \%)$ & $54(15.2 \%)$ & \\
\hline N1 & $626(60.0 \%)$ & $228(64.0 \%)$ & \\
\hline N2 & $210(20.1 \%)$ & $74(20.8 \%)$ & \\
\hline Pathological G staging & & & 0.649 \\
\hline G1 & $208(19.9 \%)$ & $71(19.9 \%)$ & \\
\hline G2 & $731(70.0 \%)$ & $243(68.3 \%)$ & \\
\hline G3 & $105(10.1 \%)$ & $42(11.8 \%)$ & \\
\hline Previous history of abdominal surgery & $98(9.4 \%)$ & $31(8.7 \%)$ & 0.782 \\
\hline
\end{tabular}


Table 2 Perioperative clinical data and postoperative long-term conditions

\begin{tabular}{|c|c|c|c|}
\hline & \multicolumn{3}{|l|}{ Before match } \\
\hline & Jejunostomy group $(n=1044)$ & Nasojejunal group $(n=356)$ & $P$ value \\
\hline Surgery time (min) & $208.8 \pm 53.5$ & $218.1 \pm 43.2$ & 0.003 \\
\hline Intraoperative indwelling time (min) & $26.6 \pm 10.4$ & $18.4 \pm 9.1$ & $<0.001$ \\
\hline Postoperative indwelling time of nutrition tube (d) & $38.6 \pm 6.9$ & $18.5 \pm 7.6$ & $<0.001$ \\
\hline Anal exhaust time (d) & $2.6 \pm 2.3$ & $3.0 \pm 1.3$ & 0.066 \\
\hline Hospitalization expense (ten thousand yuan) & $5.3 \pm 2.4$ & $5.6 \pm 4.0$ & 0.103 \\
\hline Postoperative hospital stay (d) & $14.8 \pm 5.8$ & $15.5 \pm 6.1$ & 0.088 \\
\hline \multicolumn{4}{|l|}{ Perioperative complications ( $\mathrm{n}$ ) } \\
\hline Pulmonary infection & $178(17.0 \%)$ & $79(22.2 \%)$ & 0.037 \\
\hline lleus & $18(1.7 \%)$ & $1(0.3 \%)$ & 0.042 \\
\hline Abdominal infection & $2(0.2 \%)$ & $2(0.6 \%)$ & 0.258 \\
\hline Anastomotic fistula & $36(3.5 \%)$ & $15(4.2 \%)$ & 0.616 \\
\hline Perioperative death & $1(0.1 \%)$ & $0(0.0 \%)$ & 0.559 \\
\hline Incision infection & $2(0.2 \%)$ & $4(1.1 \%)$ & 0.020 \\
\hline \multicolumn{4}{|l|}{ Tubule-related complications ( $\mathrm{n}$ ) } \\
\hline Gastrointestinal/nasal hemorrhage & $11(1.1 \%)$ & $4(1.1 \%)$ & 0.912 \\
\hline Nutrient tube slippage & $2(0.2 \%)$ & $18(5.1 \%)$ & $<0.001$ \\
\hline Intestinal fistula & $8(0.8 \%)$ & $0(0.0 \%)$ & 0.098 \\
\hline Nutrient reflux & $1(0.1 \%)$ & $20(5.6 \%)$ & $<0.001$ \\
\hline \multicolumn{4}{|l|}{ Postoperative long-term complications (> $3 \mathrm{~m}, \mathrm{n}$ ) } \\
\hline lleus & $18(1.7 \%)$ & $1(0.3 \%)$ & 0.042 \\
\hline
\end{tabular}

postoperative anal exhaust time, and postoperative hospital stay between the two groups (all $P>0.05$ ). There were also no significant differences between the two groups regarding gastrointestinal/nasal hemorrhage, abdominal infection, intestinal fistula, anastomotic fistula, and perioperative mortality rates (all $\mathrm{P}>0.05$ ). Postoperative pulmonary infection ( $17.0 \%$ vs $22.2 \%)$, incision infection $(0.2 \%$ vs $1.1 \%)$, nutrient tube slippage $(0.2 \%$ vs $5.1 \%)$ and nutrient reflux $1(0.1 \%$ vs $5.6 \%)$ rates were reduced in the Jejunostomy group compared with the Nasojejunal group $(P<0.05)$. In all, there were 18 cases with nutrient tube slippage after surgery in the Nasojejunal group, and all reported extubation without autonomous consciousness at night; tube indwelling was performed again under fluoroscopy after extubation. There was 1 patient with sudden acute cardiopulmonary arrest in the Jejunostomy group, who was transferred to the ICU for further treatment after successful rescue; the patient was discharged upon request from family members.

Meanwhile, ileus rates perioperatively ( $1.7 \%$ vs $0.3 \%)$ and at 3 postoperative months ( $1.7 \%$ vs $0.3 \%)$ were both higher in the Jejunostomy group compared with the Nasojejunal group. Precisely, there were 18 patients with postoperative ileus in the Jejunostomy group; all those with incomplete ileus who had a previous history of abdominal surgery were improved after parenteral nutrition support and discontinuation of nasal feeding. There were 18 cases with ileus 3 months after surgery, including 17 who had incomplete ileus and were improved after conservative treatment; 1 case was improved after separation of intestinal adhesion.

\section{Discussion}

In this study, operation time was shorter in the Jejunostomy group compared with the Nasojejunal group, while intraoperative and postoperative indwelling times of nutrition tubes were prolonged, demonstrating superior treatment effects for jejunostomy; meanwhile, adverse effects were generally less frequent after jejunostomy.

Further development of surgical treatment in esophageal carcinoma aims to reduce surgical trauma, and improve postoperative quality of life, as well as short- and long-term efficacies. Postoperative EN in patients with esophageal carcinoma plays an important role in maintaining normal body metabolism, functional recovery, reducing complications, and hospitalization expenses $[8$, 20]. With continuous improvement of laparoscopic equipment and techniques, laparoscopic jejunostomy has also been gradually introduced in minimally invasive esophagectomy in recent years. Matching the apparatus 
and nutrition tubes in jejunostomy would certainly increase the economic burden to some extent. In response, our center has performed laparoscopic jejunostomy since 2015, and routine laparoscopic equipment and common nutrition tubes have been used, which has reduced hospitalization expenses while avoiding excessive dependence on special apparatus in further popularization. In the present study, 1400 patients undergoing minimally invasive esophagectomy were assessed, and jejunostomy had the advantages of reducing postoperative pulmonary infection, nutrient tube slippage, nutrient reflux, and incision infection. Although the incidence of perioperative ileus and postoperative long-term ileus were slightly higher than those of the Nasojejunal group, they were relatively low, which was acceptable.

The above data showed that the Jejunostomy group was superior to the Nasojejunal group in terms of surgery time, postoperative indwelling time of the nutrition tube, postoperative pulmonary infection, and postoperative incision infection, although ileus rates were relatively higher. Zhao Song et al. [21] conducted a retrospective comparative analysis of different EN methods in 128 patients with esophageal carcinoma, and reported prolonged surgery time and postoperative indwelling time of the nutrition tube in the jejunostomy group compared with the nasojejunal group, indicating that jejunostomy improves the nutritional status of patients in a better way. The incidence of postoperative complications $(7.69 \%)$ in the jejunostomy group was significantly higher than that of the nasojejunal group (26.31\%), and it was considered that the nasointestinal tube stimulated the nasopharynx, causing difficulty in coughing, expectoration and intestinal nutrient reflux, which are main factors exacerbating pulmonary infections. Wang et al. [22] analyzed the clinical data of 28 patients with esophageal carcinoma administered laparoscopic jejunostomy, showing that the nasointestinal tube easily results in nutrient reflux, causing vomiting and aspiration pneumonia, with poor patient tolerance. Meanwhile, the advantages of a jejunostomy tube are as follows: it can be carried out a long time after surgery; when complications, such as anastomotic fistula, occur, the patients can receive long-term EN to improve prognosis; patients have high tolerance with no obvious foreign body sensation, resulting in limited effects on daily life activities. Rong Baolin et al. [23] analyzed the clinical data of 279 patients with esophageal carcinoma administered laparoscopic jejunostomy at an earlier period in our hospital, and suggested a total incidence of postoperative complications of $20.1 \%$, including 4 patients with incomplete ileus, 1 with complete ileus 3 months after surgery, and 1 with angulated torsion of the intestinal tube in the jejunostomy region shown in secondary surgical exploration, who recovered after releasing the intestinal adhesion. It is believed that the Nasojejunal group achieves cervical anastomosis, and the incidence of damage to the recurrent laryngeal nerve is higher compared with that of intrathoracic anastomosis; in addition, the nasointestinal tube is indwelt through the nose, causing cough, expectoration, nausea, and reflux, thus increasing the incidence of pulmonary infection to some extent [13]. Meanwhile, all incisions in the Jejunostomy group are of laparoscopic nature, and small auxiliary incisions are present at the upper abdomen and neck in the Nasojejunal group, so the incidence of incision infection is relatively high. In addition, a jejunostomy tube looks better and is more convenient than a nasointestinal tube, avoiding negative effects of a nasointestinal tube on social function after discharge and reducing feelings of inferiority caused by the disease.

Although the incidence of postoperative ileus was higher after jejunostomy than in the Nasojejunal group, the overall rate was not very high, and the involved patients had a history of abdominal surgery. It is recommended that patients with a previous history of abdominal surgery should be treated with tube feeding only after confirming no ileus by abdominal imaging and patients with postoperative abdominal distension and pain should be further examined. Nasal feeding should be discontinued for those with postoperative ileus, avoiding further aggravation of their condition.

Tubule-related complications are among the main evaluation factors for postoperative EN, which mainly include intestinal fistula, nutrient tube slippage, tube blocking, and ileus $[13,15,24]$. The jejunostomy tube reported in most studies has a relatively large diameter. Although this decreases the rate of tube blockage, other tubule-related complications can increase. Zhou et al. [24] analyzed the clinical data of 42 patients with esophageal carcinoma between January 2014 and July 2015, and the main tubule-related complication after jejunostomy was intestinal fistula, which may be due to loose knotting of the purse string. Postoperative care after jejunostomy was very important in preventing tube slippage and blocking. Sun Ming et al. [13] retrospectively analyzed EN in 90 patients undergoing esophagectomy through two incisions at the right chest and abdomen, showing the nasojejunal group had different degrees of nasopharyngeal discomfort; in addition, incidence of tube slippage and blocking were significantly lower in the jejunostomy group compared with the nasojejunal group. This demonstrates that although nasal feeding via a nasointestinal tube is simple, easy, and suitable for short-term feeding, it has certain shortcomings: it may damage the anastomotic stoma or stump during tube indwelling, which may easily cause intraoperative contamination; some patients have nutrient reflux 
during nasal feeding, easily resulting in lung infection and anastomotic contamination; the nasointestinal tube is generally fixed with tape and rope, and some patients may remove it due to nasopharyngeal discomfort, leading to nutrient tube slippage. Gong et al. [15] analyzed tubule-related complications in 379 patients with esophageal carcinoma administered jejunostomy between 2005 and 2013, showing that approximately $13-40 \%$ of individuals had tubule-related complications, mainly including swelling and infection around the jejunostomy area, intestinal fistula, and tube blocking; therefore, they recommended properly reducing the proportion of jejunostomy for patients with early stage of tumor and no need for further treatment after surgery, and postoperative EN treatment via a nasointestinal tube instead. While jejunostomy can be performed in patients with advanced-stage tumors, and long-term indwelling would improve the nutritional status. However, it is important to note that the diameters of the jejunostomy and nasointestinal tubes reported in the above studies were different, with certain effects on the results of postoperative tubule-related complications. In the present study, patients in the two groups were treated with Flocare $\mathrm{CH}$ 10-130 nutrition tube (Nutricia pharmaceutical co. LTD, Wuxi, China). There was no significant difference in the rate of tube blocking between the two groups, which may be predominantly related to product information, education, and nursing regarding the nutrition tube. The incidence of intestinal fistula and ileus in the Jejunostomy group were higher than those of the Nasojejunal group. Meanwhile, the Jejunostomy group showed lower nutrient reflux and nutrient tube slippage rates than the Nasojejunal group. This indicates that although the nasointestinal tube was used for a long time in esophagectomy with high safety, tubule-related complications were relatively high, and patient comfort was poor; therefore, it was mainly applied for EN after McKeown surgery. With the widespread application of minimally invasive esophagectomy in recent years, especially the popularity of minimally invasive Ivor-Lewis surgery, a traditional nasointestinal tube is no longer suitable for laparoscopic surgery and laparoscopic jejunostomy is more suitable for minimally invasive IvorLewis surgery. Although certain surgical trauma and postoperative complications will occur, most complications could be further prevented and avoided with the accumulation of related experience.

This study had several limitations. It was a singlecenter retrospective trial with a certain bias in case selection. For example, we could not perform a propensity-score matched analysis to compare the outcomes between the two matched groups because too many patients in the Nasojejunal group would have to be removed from the analysis because of the differences in cancer location between the groups. Although clinical effectiveness was significant, multi-center prospective studies are still needed for further investigation. The patient's subjective tolerance conditions were not very detailed in the medical records, including nasopharyngeal discomfort, abdominal discomfort, nausea, vomiting, and number of extubations due to intolerance. Therefore, this study could not assess differences between the two enteral nutrition methods in that respect. In addition, patients used several types of enteral nutrients, including enteral nutrition suspensions and our inhouse configured nutrients. Furthermore, this study failed to analyze nutrient differences between the two groups, because data of albumin and hemoglobin levels before surgery and at discharge were incomplete. Most importantly, patients in the two groups had different surgical methods. However, for the benefit of patients, the choice of the EN method would be affected by the surgical technique, so the influence of the surgical method on the above results could not be completely eliminated.

\section{Conclusion}

In conclusion, jejunostomy is safe and reliable in the treatment of thoracic segment esophageal carcinoma during complete thoracoscopic and laparoscopic IvorLewis esophagectomy. Compared with a nasointestinal tube in minimally invasive McKeown surgery, it has the advantages of reducing postoperative pulmonary infection, nutrient tube slippage, nutrient reflux, and incision infection. Although the incidence of perioperative ileus and postoperative long-term ileus were slightly higher than those of the Nasojejunal group, the overall rates were relatively low and within an acceptable range. Further accumulation of related experience and techniques would help prevent and treat these postoperative complications in the future.

\section{Abbreviations}

NCCN: National Comprehensive Cancer Network; EN: enteral nutrition

\section{Acknowledgements \\ None.}

\section{Authors' contributions}

Xiangxiang Sun carried out the studies, participated in collecting data, and drafted the manuscript. Ran Xion performed the statistical analysis. Xinyu Mei and Mingfa Guo participated in its design. Jieyong Tian participated in acquisition, analysis, or interpretation of data and draft the manuscript. All authors read and approved the final manuscript.

\section{Funding}

None.

\section{Availability of data and materials}

The datasets used and/or analyzed during the current study are available from the corresponding author on reasonable request. 


\section{Ethics approval and consent to participate}

The study protocol was approved by the Ethics Committees of the First Affiliated Hospital of University of Science and Technology of China, Division of Life Sciences and Medicine, and all participants provided written informed consent.

\section{Consent for publication}

Not applicable.

\section{Competing interests}

All authors declare that they have no conflict of interests.

Received: 12 December 2019 Accepted: 18 May 2020

Published online: 17 June 2020

\section{References}

1. Domper Arnal MJ, Ferrández Arenas Á, Lanas AÁ. Esophageal cancer: risk factors, screening and endoscopic treatment in Western and eastern countries. World J Gastroenterol. 2015;21(26):7933-43.

2. Pennathur A, Gibson MK, Jobe BA, Luketich JD. Oesophageal carcinoma. Lancet (London, England). 2013;381(9864):400-12.

3. Huang F-L, Yu S-J. Esophageal cancer: risk factors, genetic association, and treatment. Asian J Surg. 2018;41(3):210-5.

4. Tan LJ, Shen YX. Minimally invasive esophagectomy-technique and experience of Zhongshan hospital Fudan University. J Clin Surg. 2016; 24(7):557-9.

5. Yang Q, Wang YX, He M, Li J, Qi Z, Zhu SC, et al. Factors affecting on longtime survival in patients with stage III thoracic esophageal carcinoma after esophagectomy. Zhonghua zhong liu za zhi. 2016;38(7):530-7.

6. Berry MF. Esophageal cancer: staging system and guidelines for staging and treatment. J Thorac Dis. 2014;6(Suppl 3):S289-S97.

7. Yasuda IL, Rufo PA. Protein-Losing Enteropathy in the Setting of Severe Iron Deficiency Anemia. J Investig Med High Impact case Rep. 2018;6: 2324709618760078

8. Wang J, Zhi LI, Zhen F, Zhang J, Luo J. Feeding tube placement and postoperative nutritional support for esophageal carcinoma patients. Chin J Clin Oncol. 2014:41(23):1503-5.

9. Zhang ZH, Ge JM, Y. Y, et al. Application of laparoscopic jejunum pull through ostomy in nutritional support after thoracolaparoscopic resection of esophageal carcinoma. Chin J Minim Inva Surg. 2017;17(10):936-8.

10. Peng JS, Li Y. Choice of nutritional plans for patients with gastrointestinal neoplasms. Chin J Gastrointest Surg. 2012;15(5):437-9.

11. Lis CG, Gupta D, Lammersfeld CA, Markman M, Vashi PG. Role of nutritional status in predicting quality of life outcomes in cancer--a systematic review of the epidemiological literature. Nutr J. 2012;11:27.

12. de Aguilar-Nascimento JE, Bicudo-Salomao A, Portari-Filho PE. Optimal timing for the initiation of enteral and parenteral nutrition in critical medical and surgical conditions. Nutrition. 2012;28(9):840-3.

13. Sun M, Yu B, Liu JH, et al. Application of jejunostomy in radical operation of esophageal carcinoma through right thoracotomy and abdominal incision. Chin J Postgrad Med. 2016;39(6):504-6.

14. Li H, Hu B, You B. Miao J-b, Fu Y-I, Chen Q-r. combined laparoscopic and thoracoscopic Ivor Lewis esophagectomy for esophageal cancer: initial experience from China. Chin Med J. 2012;125(8):1376-80.

15. Gong L, Ma MQ, Jiang $H J$, et al. Exploration of standardized enteral nutrition therapy through jejunostomy after esophagectomy. In: 2015 Chinese International Symposium of Nutritional Oncology (CISNO), the 3rd National Conference on Cancer Nutrition and Supportive Care (NCCNSC) \& the 1st Cross-Strait Cancer Nutrition Summit Symposiums; 2015. p. 384-9.

16. Marano L, D'Ignazio A, Cammillini F, Angotti R, Messina M, Marrelli D, et al. Comparison between 7th and 8th edition of AJCC TNM staging system for gastric cancer: old problems and new perspectives. Transl Gastroenterol Hepatol. 2019;4:22

17. D'Amico TA. Mckeown esophagogastrectomy. J Thorac Dis. 2014;6(Suppl 3): S322-S4.

18. Lozac'h P, Topart P, Etienne J, Charles JF. Ivor Lewis operation for epidermoid carcinoma of the esophagus. Ann Thorac Surg. 1991;52(5): 1154-7.

19. Allen MS. Ivor Lewis esophagectomy. Semin Thorac Cardiovasc Surg. 1992; 4(4):320-3.
20. Chen W, Xu SC, Feng QL. Analysis of occurrence of aspiration pneumonia in critical ill patients under two jejunum nutrition administration approaches. Chin J Digest Endosc. 2014;31(2):162-3.

21. Zhao S, Wu B, Qi Y, et al. Application of jejunostomy and nasojejunal nutrition tubes in esophagectomy. Chin J Gerontol. 2014;34(10):2740-1.

22. Wang $Y H$, Hu X, Zhang RQ, et al. The feasibility of application of needlecatheter jejunostomy under combined thoracoscopic and laparoscopic esophagectomy. Chin J Thorac Cardiovasc Surg. 2016;32(10):638-9.

23. Rong BL, Mei XY, Wu XN. The application of laparoscopic jejunostomy during complete thoracoscopic and laparoscopic Ivor-Lewis esophagectomy. Chin J Clin Healthcare. 2017:20(2):170-2.

24. Zhou ZL, Li JF, Li ZX, et al. Laparoscopic needle catheter jejunostomy in the minimally invasive esophagectomy. Chin J Thorac Cardiovasc Surg. 2016; 32(12):767-8.

\section{Publisher's Note}

Springer Nature remains neutral with regard to jurisdictional claims in published maps and institutional affiliations.
Ready to submit your research? Choose BMC and benefit from:

- fast, convenient online submission

- thorough peer review by experienced researchers in your field

- rapid publication on acceptance

- support for research data, including large and complex data types

- gold Open Access which fosters wider collaboration and increased citations

- maximum visibility for your research: over $100 \mathrm{M}$ website views per year

At BMC, research is always in progress.

Learn more biomedcentral.com/submissions 